\title{
Application of 3D Visualization Technology in Complex Abdominal Wall Defects
}

\author{
Zhicheng Song ${ }^{1,2, *}$ \\ Wenpei Dong ${ }^{1,2, *}$ \\ Dongchao Yang ${ }^{2}$ \\ Jianjun Yang ${ }^{2}$ \\ Jugang $\mathrm{Wu}^{2}$ \\ Yiping Wang ${ }^{2}$ \\ Yan $\mathrm{Gu}^{1,2}$ \\ 'Department of General Surgery, \\ Huadong Hospital, Fudan University, \\ Shanghai, People's Republic of China; \\ ${ }^{2}$ Department of General Surgery, Hernia \\ and Abdominal Wall Surgery Center of \\ Shanghai Jiao Tong University, Shanghai \\ Ninth People's Hospital, Shanghai Jiao \\ Tong University School of Medicine, \\ Shanghai, People's Republic of China \\ *These authors contributed equally to \\ this work
}

Correspondence: Yan Gu

Department of General Surgery, Huadong Hospital, Fudan University, No. 22I West Yan'an Road, Shanghai, 200040, People's

Republic of China

Email yangu@shsmu.edu.cn
Purpose: To explore the value of medical three-dimensional visualization technology in precise preoperative assessment of complex abdominal wall defects.

Methods: The clinical data of 30 patients were analyzed retrospectively from November 2017 to December 2020 in our department. Ten patients had abdominal wall hernias and 20 patients suffered from abdominal wall tumors. CT examination was performed, and data were stored in the form of DICOM. Three-dimensional reconstruction and related data analysis were performed by Medraw software, which can accurately show the calculation of the abdominal wall defect area, abdominal wall defect classification and zoning.

Results: The ratio of the volume of the hernia sac to the whole abdominal volume in 10 patients with abdominal wall hernia was $4.75 \%$. The average ratio of defect area to the whole abdominal wall in 16 patients suffered from abdominal wall tumors was $17.68 \%$. Preoperative three-dimensional reconstruction can accurately obtain an average abdominal wall defect area of $227.83 \pm 157.33 \mathrm{~cm}^{2}$ and accurate abdominal wall classification and zoning. Combined with clinical information, we can develop personalized surgical plans for patients. The average operating time was $5.39 \pm 2.71 \mathrm{~h}$, respectively, and the average hospital stay was $22.77 \pm 11.59$ days. The mean follow-up time was $21.09 \pm 9.72$ months. The incidence of postoperative complications was $23.33 \%(7 / 30)$. The recurrence rates of incisional hernias and abdominal wall tumors were $20.00 \%(2 / 10)$ and $15.00 \%(3 / 20)$, respectively. The patient survival rate was $86.67 \%(26 / 30)$.

Conclusion: Three-dimensional visualization technology can be used for the accurate evaluation of patients with complex abdominal defects before surgery and can help surgeons design personalized surgical plans for patients.

Keywords: three-dimensional visualization, complex abdominal wall defects, abdominal wall tumor, abdominal wall reconstruction

\section{Introduction}

Computer technology has made digital medicine represented by artificial intelligence an important direction of medical development in the 21st century. In 2012, Lambin proposed the concept of radiomics, which refers to the high-throughput and automatic quantitative analysis of CT, MRI, PET and other large-scale imaging data through machine learning to achieve tumor segmentation, feature extraction and model establishment. ${ }^{1}$ However, these two-dimensional imaging methods cannot provide clinicians with intuitive, stereoscopic, and omnidirectional images to reflect the relationship between the tumor and adjacent organs. ${ }^{2}$ Deeper prediction and analysis are performed to transform the image information into deep-level disease features to 
assist physicians in making accurate diagnoses. As a new type of computer-assisted imaging technology, threedimensional visualization (3DV) exhibits accurate, intuitive and clear three-dimensional images through powerful postprocessing functions and helps surgeons assess surgical risks, design surgical planning and simulate surgery and intraoperative navigation. ${ }^{3} 3 \mathrm{DV}$ was rapidly developed in many medical fields and applied to clinical departments, such as orthopedics, general surgery, and neurosurgery. ${ }^{4-7}$ With the help of 3DV, it was more intuitive for surgeons to sense the anatomy of diseases, and to develop personalized treatment plans for patients. ${ }^{8}$ However, there have been no published reports on the use of $3 \mathrm{DV}$ for complex abdominal wall defects. The aim of this study was to evaluate the value of $3 \mathrm{DV}$ for reconstructing complex abdominal wall defects.

\section{Patients and Methods}

\section{Patient Information}

From November 2017 to December 2020, clinical data of 30 patients with complex abdominal wall defects were collected from the Department of General Surgery, Shanghai Ninth People's Hospital, Shanghai Jiao Tong University School of Medicine. The mean age of the patients was $51.27 \pm 19.02$ years, and the mean score of American society of anesthesiologists (ASA) physical status classification system was $1.97 \pm 0.67 ; 10$ patients had abdominal wall hernias, and 20 patients had abdominal wall tumors. All patients signed an informed consent form before surgery. The study was approved by the Ethics Committee of Shanghai Ninth People's Hospital (SH9H-2020-TK376-1). This study was conducted in accordance with the declaration of Helsinki.

\section{Preoperative CT Examination}

All patients underwent total abdominal enhanced CT with a layer thickness of $1 \mathrm{~mm}$. The image information was stored in the format of Digital Imaging and
Communications in Medicine (DICOM) and imported into Medraw software for 3D reconstruction.

\section{Three-Dimensional Reconstruction and Surgical Planning}

The image data were imported into Medraw software (Image Medraw Technology Co., Ltd., China) in DICOM format for three-dimensional reconstruction (Figure 1). The radiologists and clinicians jointly built a threedimensional model that included bone, muscle, blood vessels, urinary system and abdominal wall tumor lesions and provided corresponding records and statistics (Figure 2). The combination, disassembly, rotation and other methods are used to show the anatomical relationship between abdominal wall tumors and surrounding important organs and provide theoretical support for the preparation of individualized surgical plans. Combined with the classification and division of the abdominal wall, the type of abdominal wall defect is determined. ${ }^{9}$ Preoperative accurate judgment and the development of individualized treatment plan were also made.

\section{Enrollment and Exclusion Criteria}

Size and Location

The large-sized abdominal wall hernia including parastomal, lumbar, lateral and subcostal locations of hernias had a larger width $\geq 10 \mathrm{~cm}^{10}$ And the loss of domain was $\geq 20 \%$.

\section{Contamination and Soft Tissue Condition}

The contaminated or dirty wound environment of patients was a significant factor for enrolled consideration. And the soft tissue condition could be divided into full-thickness abdominal wall defects, loss of substance, distorted anatomy, denervated muscles, skin grafts, wound ulcers, open abdomen, and presence of enterocutaneous fistula.
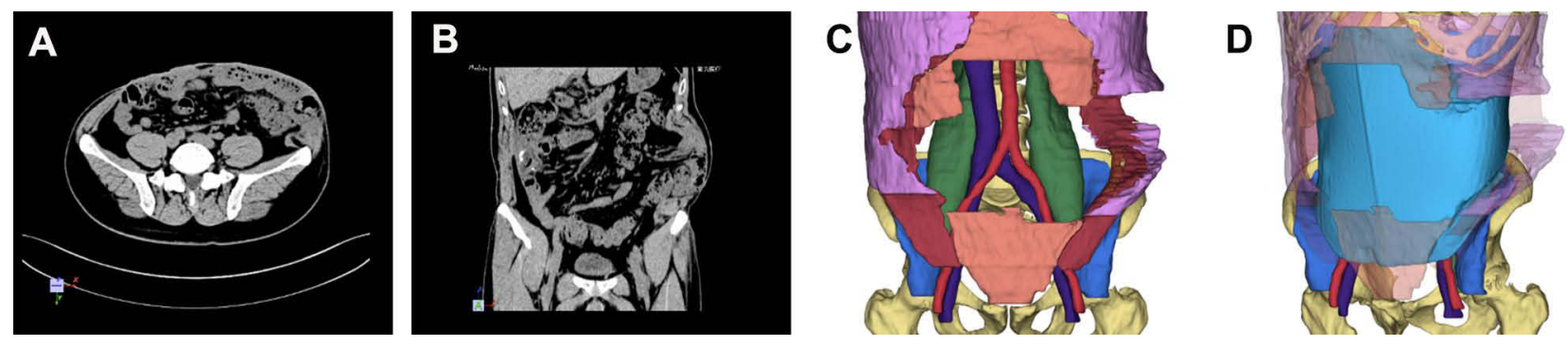

Figure I CT examination and three-dimensional reconstruction of incisional hernia. (A) Transverse section examination of CT. (B) Coronal section examination of CT. (C) Abdominal wall defect. (D) Three-dimensional reconstruction of incisional hernia with mesh. 

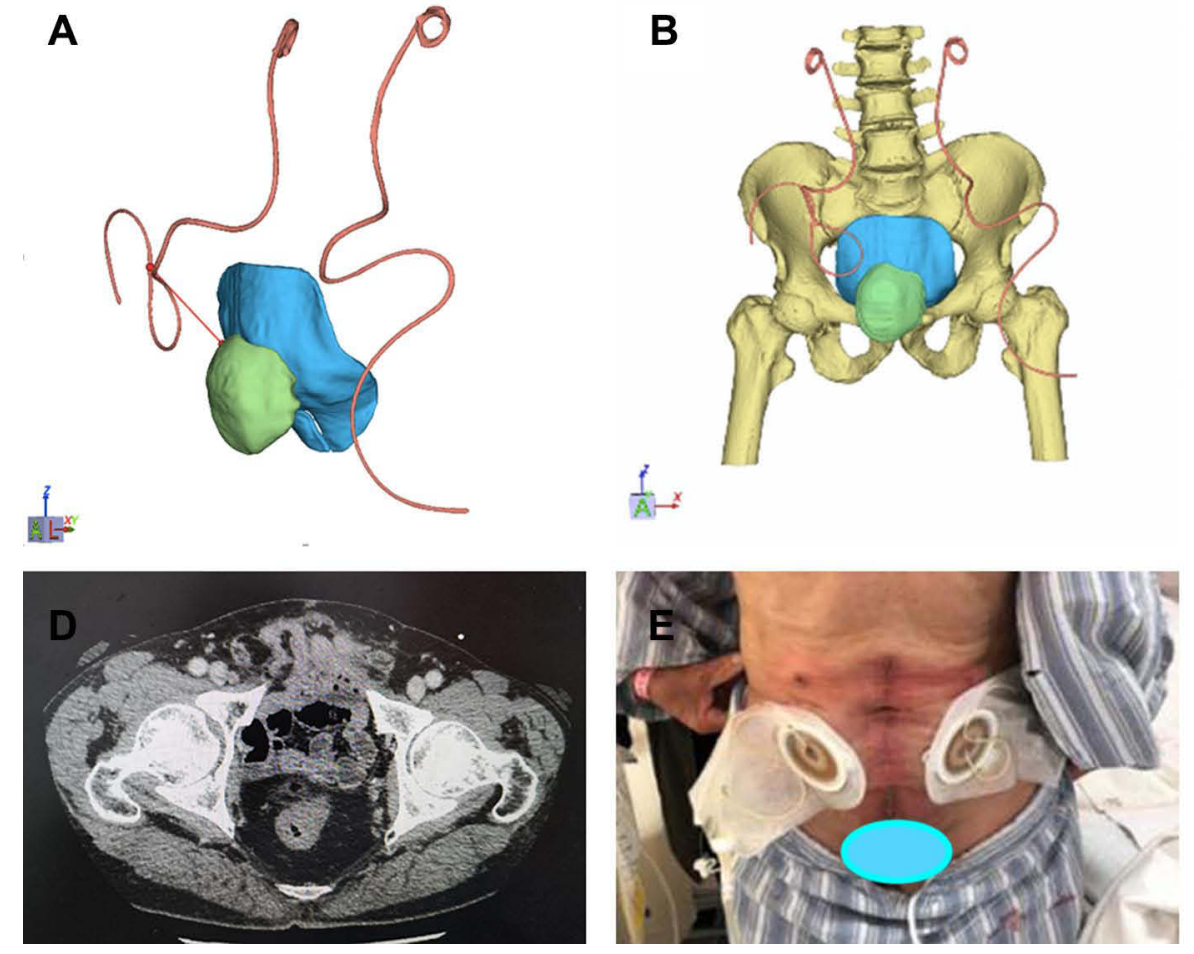

Figure 2 Three-dimensional reconstruction of suprapubic hernia of cystectomy patients with abdominal wall ureterostomy. (A) Distance between right ureteral catheter and tumor. (B) The relationship between tumor, ureteral catheters and mesh. (C) Distance between right ureteral catheter and tumor. (D) Transverse section examination of CT. (E) Abdominal wall.

\section{Patient History and Risk Factors}

Patients suffered from complex abdominal wall defects might have a recurrent hernia or risk factors such as obesity, diabetes, old age, steroid use, or poor nutritional state (albumin $<30 \mathrm{~g} / \mathrm{dL}$ ) that impair wound healing. And increased intra-abdominal pressure, previous wound dehiscence, or previous mesh infection should also be taken into consideration.

\section{Clinical Situation}

Emergency operation with bowel resection, intraperitoneal mesh removal, multiple hernia defects, and no primary closure possible without component separation were also diagnostic factors for complex abdominal wall defects.

\section{Observation Indicators}

Three-dimensional reconstruction accurately calculates the area of the proposed abdominal wall defect and the classification and division of the abdominal wall defect; comprehensive consideration of various factors, creating an individualized surgical plan for the patient; statistics of intraoperative and postoperative complications.

\section{Follow-Up}

Follow-up was performed in outpatient and telephone ways to understand the postoperative complications of patients. The follow-up period was December 2020.

\section{Statistical Analysis}

Application SPSS 22.0 statistical software analysis. Measurement data for normal distributions are expressed as the mean \pm standard deviation.

\section{Results}

\section{General Situation}

According to the three-dimensional reconstruction model before surgery, 10 patients underwent reconstruction of abdominal wall defects, and 20 patients underwent radical tumor resection and reconstruction. The abdominal wall defect area was $227.83 \pm 157.33 \mathrm{~cm}^{2}$. The defects were accurately typed and partitioned (Table 1); the average operating time was $5.39 \pm 2.71 \mathrm{~h}$, respectively, and the average hospital stay was $22.77 \pm 11.59$ days. The mean follow-up time was $21.09 \pm 9.72$ months. The number of abdominal wall surgeries was $1.60 \pm 1.06$. The incidence of postoperative complications after abdominal wall 


\begin{tabular}{|c|c|c|c|}
\hline ن. & 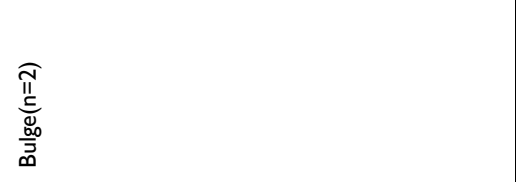 & 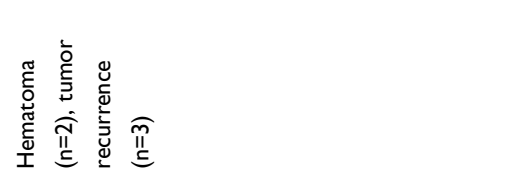 & \\
\hline 吉离 & 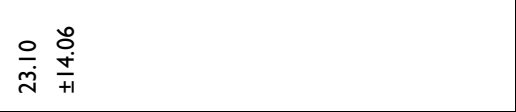 & 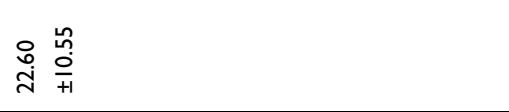 & 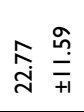 \\
\hline 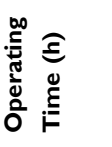 & 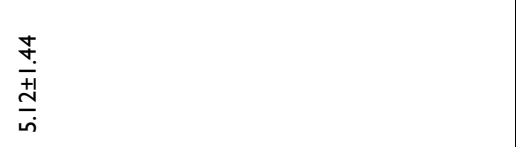 & 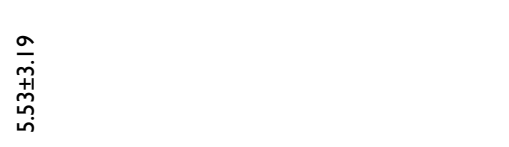 & 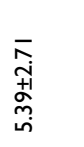 \\
\hline 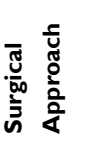 & 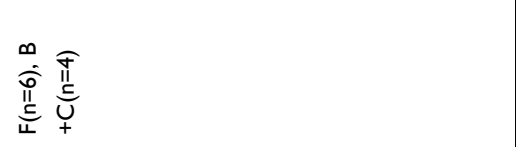 & 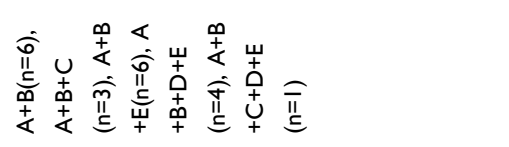 & \\
\hline 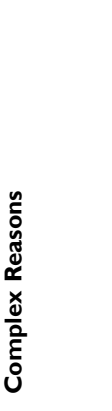 & 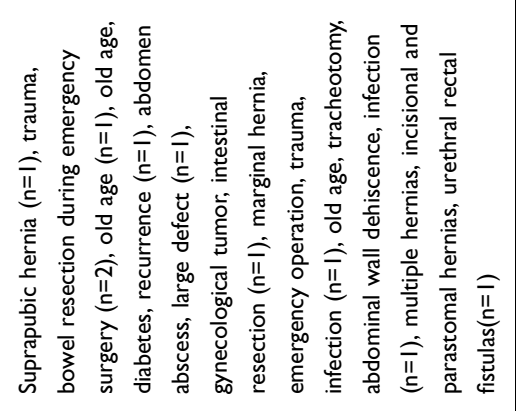 & 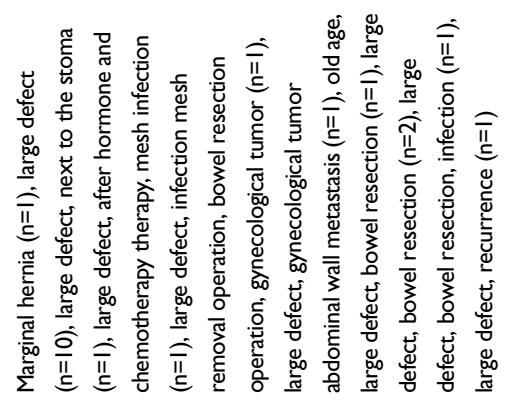 & \\
\hline N⿳亠口冋! & 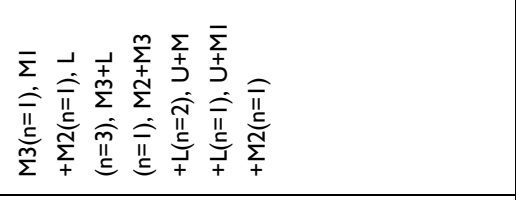 & 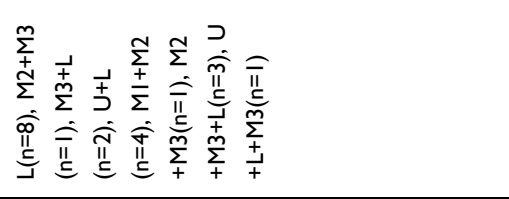 & \\
\hline 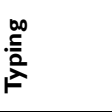 & $\underset{=}{\stackrel{0}{\frac{0}{11}}}$ & 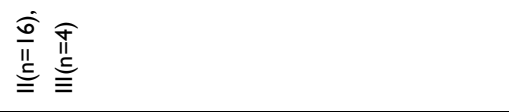 & \\
\hline 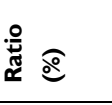 & 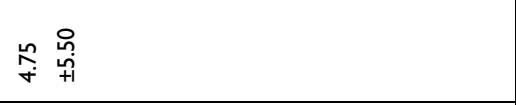 & 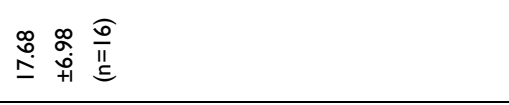 & \\
\hline 苞导 & $\begin{array}{ll}0 & 0 \\
0 & 0 \\
\infty & \infty \\
\frac{\infty}{N} & \stackrel{\infty}{+1}\end{array}$ & 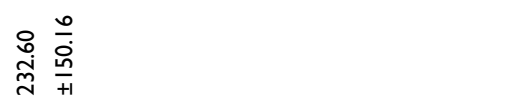 & 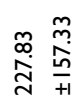 \\
\hline 童亮 & 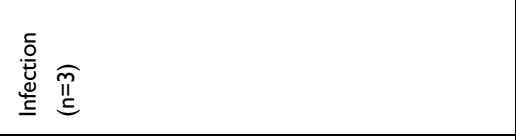 & 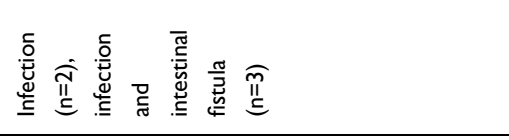 & \\
\hline $\begin{array}{l}\overleftarrow{4} \\
\text { पू }\end{array}$ & 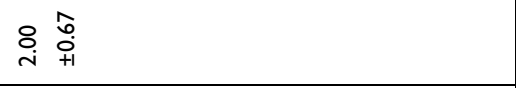 & 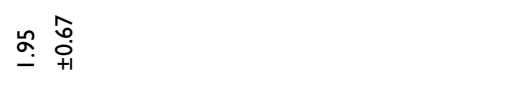 & s. \\
\hline$\overline{\Sigma_{\infty}}$ & 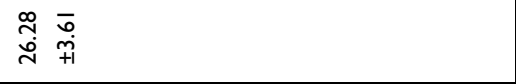 & 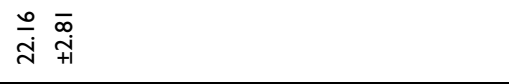 & $\begin{array}{l}\text { 芯 } \\
\stackrel{\sim}{\sim} \underset{+}{+}\end{array}$ \\
\hline 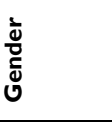 & 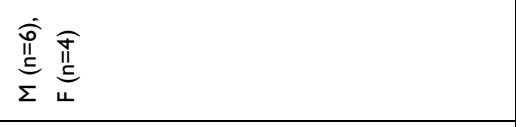 & 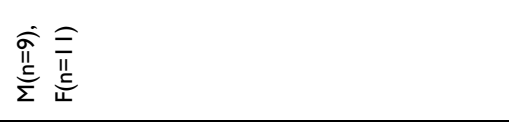 & 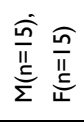 \\
\hline 嵒 & 品 & 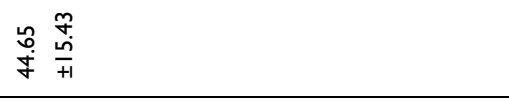 & 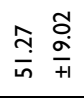 \\
\hline 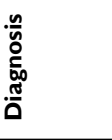 & 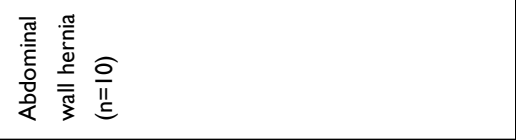 & 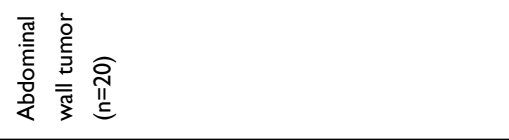 & 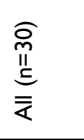 \\
\hline
\end{tabular}


surgery was approximately $23.33 \%$ (7/30). Three patients had recurrence of abdominal wall tumors, two patients had hematomas and two patients had abdominal wall bulges.

\section{Abdominal Wall Defect Types and Zones}

The three-dimensional reconstruction of the abdominal wall tumor can visually show that the tumor is located in the skin layer, fascia layer or whole skin layer. The complex abdominal wall defects were classified into three different types and three zones by the guidelines which included type I, II, and III, zone M, U and L. ${ }^{8,9,11}$ The defect were also classified from grade 1 to 4 by using the Ventral Hernia Working Group's (VHWG) grading system. $^{8}$

\section{Abdominal Wall Tumors and Adjacent Relationships Determined the Surgical Approach}

The surgical method for complex abdominal wall defects is mainly based on the type of abdominal wall defect. If the myofascial layer cannot be closed, synthetic or biological mesh + tissue structure separation technology may be used. If the defect belongs to type III, the skin flap should be transferred adjacent to the above surgical basis. If the tumor has metastasized, only palliative tumor resection should be performed. Abdominal wall tumors are less than $3 \mathrm{~cm}$ in size. Considering the origin of transverse abdominal muscles or oblique muscles, laparoscopic abdominal wall tumor resection may be considered. Threedimensional reconstruction was used to accurately assess the defect or the tumor and adjacent organs and to develop an individualized surgical plan (Table 1).

\section{The Patients Were Followed Up}

Postoperative follow-up was conducted until December 2020, with an average follow-up time of 21.09 \pm 9.72 months. The survival rate of patients was $86.67 \%$ (26/30), and the postoperative complication rate was $23.33 \%(7 / 30)$.

\section{Discussion}

Complex abdominal wall defects are technically challenging and time-consuming. Due to the varied surgeon's experience, many classification systems for the corresponding treatment have been reported..$^{8,9,12-14}$ These classification systems are based mostly on surgeon experience or involve the aid of related research or statistical analysis of patient cohorts. To date, there is no consensus as to what exactly constitutes a complex abdominal wall defect. However, four categories and three severity classes of complex abdominal wall defects, comprehensive coverage and accurate assessment are accepted by most surgeons. ${ }^{10}$ Therefore, we used this method to classify patients and accurately evaluate them preoperatively in our study.

The traditional preoperative evaluation of abdominal wall defects mainly relies on CT or MRI examination imaging data combined with the surgeon's spatial imagination and clinical experience, which is sometimes inaccurate and vague and even affects the selection of surgical methods, especially for uncertain complex cases. Threedimensional reconstruction plays an important role in the accurate assessment of abdominal wall defects and the guidance of preoperative planning. Through threedimensional reconstruction and analysis of CT data, surgeons can stereoscopically and comprehensively observe the location, shape and size of lesions to improve preoperative planning. ${ }^{4}$ The main advantages are summarized as follows. Through accurate calculation and simulation, we can calculate the area, classification and zoning; the distance to the abdominal surface landmarks and the most suitable mesh size and simulate the process of the operation. Combined with the local incision and comprehensive situation, a personalized surgical protocol is developed before the operation.

If the abdominal wall defect is caused by the tumor, the effect of three-dimensional visualization technology is more significant. It cannot only complete the above basic information, but it can also accurately calculate the size and location of the tumor and the expansion of the abdominal wall tumor resection, assess the type and division of abdominal wall defects, determine whether the tumor invades adjacent organs or distant metastases and simulate the main surgical process. In addition, it can facilitate communication between doctors and patients and alleviate the contradiction between doctors and patients to a certain extent. Regarding the relationship with adjacent organs for precise preoperative prevention, if the tumor invades the blood vessels, ureters, intestines and nerves, it is necessary to make adequate preoperative preparations for the corresponding tissue and organ resection, which is of great significance in improving the success rate of surgery and the satisfaction of doctors and patients and reducing postoperative complications. In this study, all patients were given accurate calculations, simulations, assessments, and prevention through three-dimensional reconstruction. Among them, the proportion of abdominal 
wall tumors invading surrounding tissues and organs was $60.81 \%(14 / 23)$. Visceral invasion includes the peritoneum, sacrum, pubic bone, ribs, intestines, iliac vessels, bladder and uterus. Before surgery, we develop a personalized surgical plan through multidisciplinary discussions.

For secondary abdominal wall tumors, invasion of adjacent organs, including invasion of the blood vessels, nerves, intestines, bladder and uterus, is often complicated, and the complexity of the operation is increased. All the information cannot be visually and accurately presented during the operation, and it is impossible to simulate the operation by traditional methods. Three-dimensional visualization technology can perform three-dimensional reconstruction of abdominal wall tumors, transform two-dimensional images into volume images and display the adjacent relationship between abdominal wall tumors and surrounding tissues or organs in an all-round, multiangle and transparent manner. Different splitting and combining pairs can be combined. Selective observation of specific lesions, especially the invasion of adjacent abdominal wall tumors to adjacent tissues or organs, can be rotated to observe the distance between the lesion and the intestines, blood vessels or ureters in a three-dimensional plane at multiple angles to achieve accurate preoperative prevention. Three- dimensional visualization, as a new technical method, plays an important role in preoperative evaluation and surgical planning and has been used popularly in liver and pancreas surgery. ${ }^{4}$ To date, there is no relevant research on abdominal wall tumors. In this study, three-dimensional reconstruction was used to evaluate abdominal wall tumors, which included location, size, classification and zone of abdominal wall tumors as well as adjacent tissue invasion and the presence of distant metastases. The abovementioned case summarized that the advantages of three-dimensional visualization were better reflected in cases of secondary abdominal wall tumors.

Abdominal wall tumors can be divided into primary and secondary abdominal wall tumors. Desmoid tumors, caused by the proliferation of mesenchymal stem cells that can occur in any part of the body, were the most common primary abdominal wall tumors in our study (Figure 3). Tumors in the limbs have the worst prognosis, and abdominal tumors have the best prognosis, with a local recurrence-free survival rate of $91 \%$ in 5 years. ${ }^{15}$ Incomplete surgery or trauma can stimulate cell proliferation, and the recurrence rate after traditional surgery can reach up to $50 \% .^{16}$ Therefore, enlarged resection is an important treatment method; however, its threedimensional reconstruction is generally relatively simple.
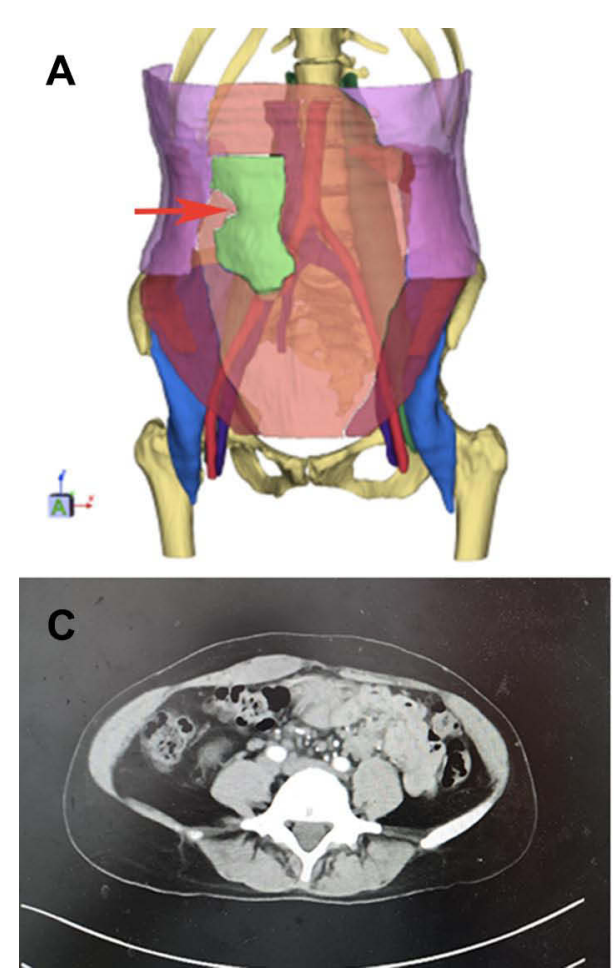
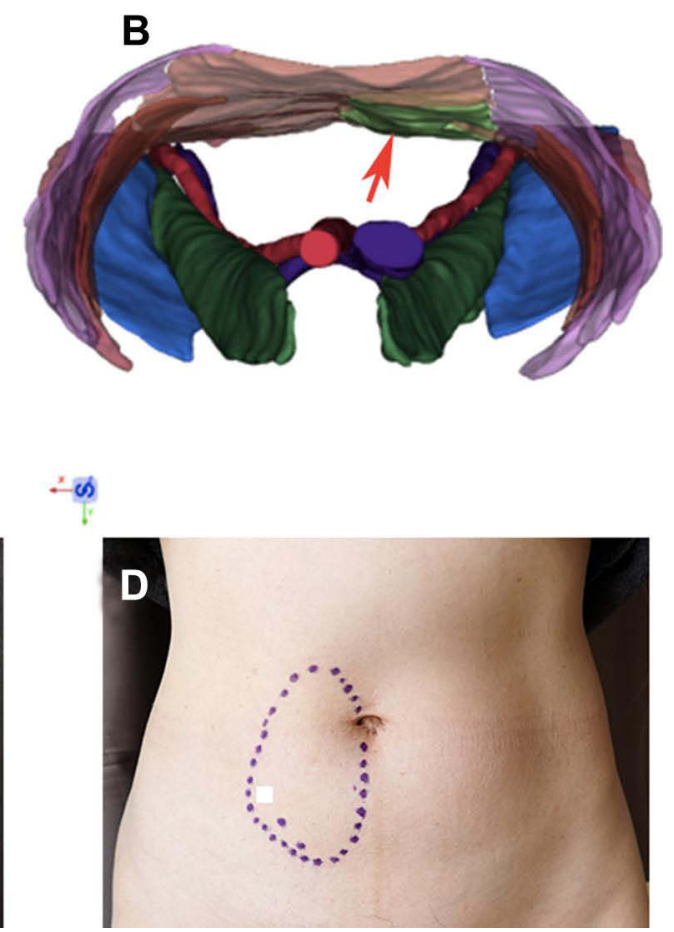

Figure 3 Primary abdominal wall tumor. (A) Three-dimensional reconstruction of tumor. Red arrow indicates tumor (green marker). (B) Three-dimensional reconstruction view from above. Red arrow indicates tumor (green marker). (C) Transverse section examination of CT. (D) Abdominal wall. 
Secondary abdominal wall tumors are more complicated, and two major factors need to be considered before surgery (Figure 4). The local condition of the abdominal wall tumor includes the location of the abdominal wall, the situation of soft tissue around the tumor and abdominal wall, with or without infection or contamination, the extent to which the abdominal organs or adjacent organs were invaded, etc. The comprehensive condition of patients is another major factor. Evaluating whether the tumor has extensive metastasis or the patient can tolerate surgery is key before surgery. Enlarged resection is the main method for the treatment of abdominal wall tumors. Regardless of the initial or multiple operations, the resection range should be sufficient. In principle, it should exceed the tumor margin by 2 to $3 \mathrm{~cm}$. In addition, it should be used in conjunction with rapid intraoperative pathological examination to ensure that the resection margin is free. For giant abdominal wall tumors or tumors in special locations, the huge abdominal wall defects often caused by enlarged resection pose a great challenge to the surgeon. Therefore, before abdominal tumor surgery, especially in patients with complex abdominal wall defects, accurate assessment of the abdominal wall tumor and its adjacent organs is required.

In summary, three-dimensional visualization can create a safe surgical resection range, accurately assess the type and division of abdominal wall defects, indirectly guide the formulation of surgical plans and avoid damage to important organs. As a new clinical disease diagnosis and treatment mode, three-dimensional visualization provides clinicians with three-dimensional, intuitive and comprehensive image information, analyzes the relationship between abdominal wall tumors and adjacent organs and provides more reliable protection for difficult abdominal wall surgery.

Based on our experience, the local abdominal wall condition for complex abdominal wall defects need precisely assessed in pre-operation. The guidelines and 3DV technology can help accurately evaluate local conditions, the type, and zone of abdominal wall defect. And 3DV can further assess the invasion of abdominal wall tumor, defect area, and the size of mesh for surgery. 3DV also avoids intraoperative and postoperative risks, helps to develop personalized
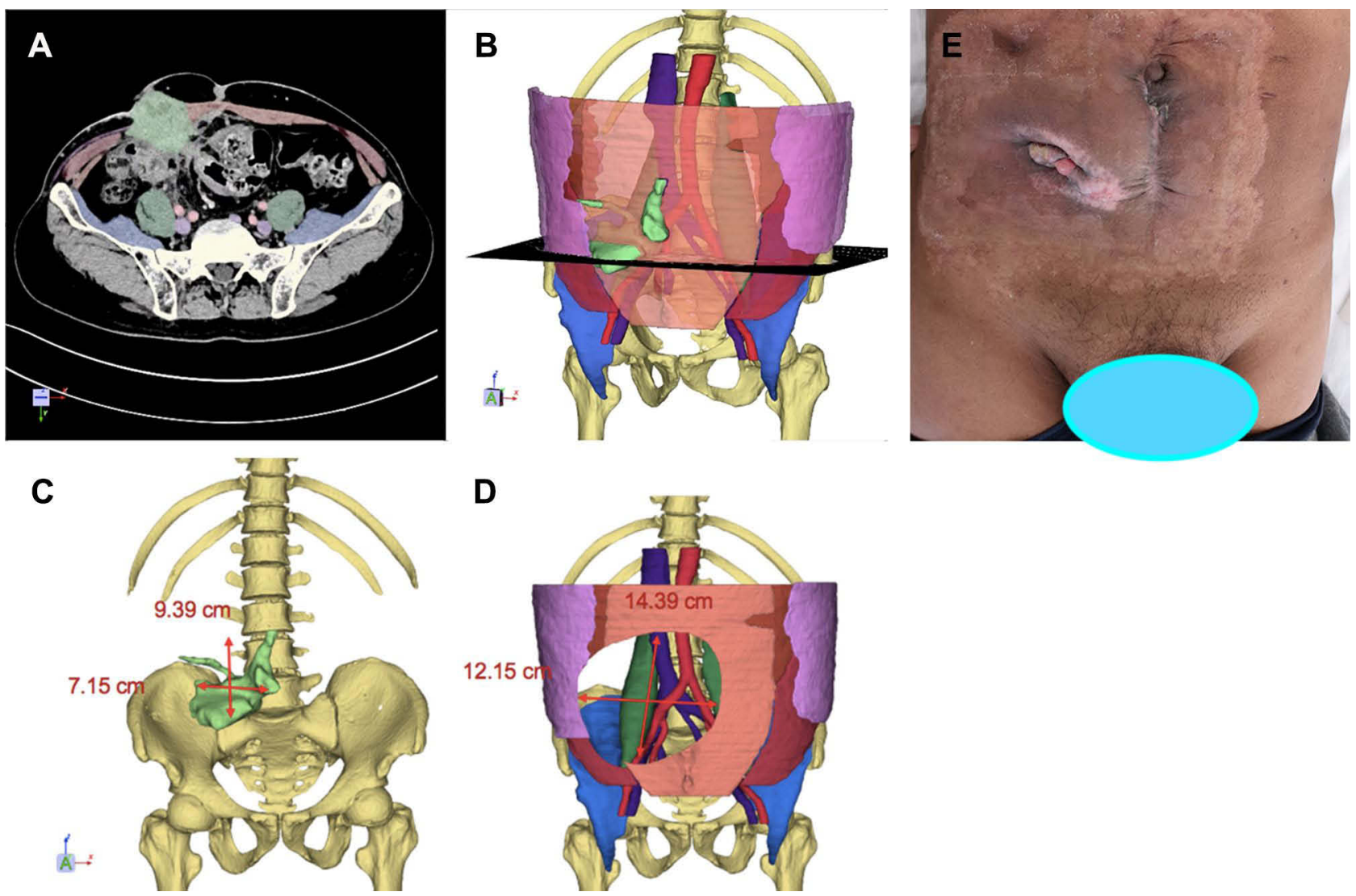

Figure 4 Secondary abdominal wall tumor. (A) Transverse section examination of CT. (B) Three-dimensional reconstruction of tumor (green marker). (C) Abdominal wall. (D) Size of tumor. (E) Abdominal wall defect. 
treatment plans for patients. However, three-dimensional reconstruction is still insufficient due to the limitations of technology. Based on image information, the boundary between nerves and surrounding soft tissue cannot be accurately distinguished. The diagnoses cannot be automatically completed by a computer and the participation of radiologists and surgeons are required.

\section{Conclusion}

Three-dimensional visualization technology can be used for accurate evaluation of patients with complex abdominal defects before surgery and help surgeons design personalized surgical plans for patients.

\section{Acknowledgments}

We thank all patients who provided the follow-up information. Zhicheng Song and Wenpei Dong contributed equally to this study and are co-first authors.

\section{Funding}

This work was supported by grants from National Natural Science Foundation of China (81970455), the Foundation of Science and Technology Commission of Shanghai Municipality (20Y11909100), Cross disciplinary Research Fund of Shanghai Ninth People's Hospital, Shanghai JiaoTong University School of Medicine (JYJC201914), and Clinical Research Project of MultiDisciplinary Team, Shanghai Ninth People's Hospital, Shanghai JiaoTong University School of Medicine (201909).

\section{Disclosure}

The authors declare that they have no conflicts of interest.

\section{References}

1. Lambin P, Rios-Velazquez E, Leijenaar R, et al. Radiomics: extracting more information from medical images using advanced feature analysis. Eur J Cancer. 2012;48(4):441-446. doi:10.1016/j. ejca.2011.11.036

2. Decazes P, Hinault P, Veresezan O, Thureau S, Gouel P, Vera P. Trimodality PET/CT/MRI and radiotherapy: a mini-review. Front Oncol. 2020;10:614008. doi:10.3389/fonc.2020.614008
3. Rowe SP, Chu LC, Fishman EK. 3D CT cinematic rendering of the spleen: potential role in problem solving. Diagn Interv Imaging. 2019;100(9):477-483. doi:10.1016/j.diii.2019.03.005

4. Cai W, Fan Y, Hu H, Xiang N, Fang C, Jia F. Postoperative liver volume was accurately predicted by a medical image three dimensional visualization system in hepatectomy for liver cancer. Surg Oncol. 2017;26(2):188-194. doi:10.1016/j.suronc.2017.03.006

5. Yang J, Tao HS, Cai W, et al. Accuracy of actual resected liver volume in anatomical liver resections guided by 3-dimensional parenchymal staining using fusion indocyanine green fluorescence imaging. J Surg Oncol. 2018;118(7):1081-1087. doi:10.1002/ jso. 25258

6. Bikis C, Degrugillier L, Thalmann P, et al. Three-dimensional imaging and analysis of entire peripheral nerves after repair and reconstruction. J Neurosci Methods. 2018;295:37-44. doi:10.1016/j. jneumeth.2017.11.015

7. Fang C, An J, Bruno A, et al. Consensus recommendations of three-dimensional visualization for diagnosis and management of liver diseases. Hepatol Int. 2020;14(4):437-453. doi:10.1007/ s12072-020-10052-y

8. Gu Y, Wang P, Li H, Tian W, Tang J. Chinese expert consensus on adult ventral abdominal wall defect repair and reconstruction. $\mathrm{Am}$ J Surg. 2020. doi:10.1016/j.amjsurg.2020.11.024

9. Tang R, Gu Y, Gong DQ, Qian YL. Immediate repair of major abdominal wall defect after extensive tumor excision in patients with abdominal wall neoplasm: a retrospective review of 27 cases [corrected]. Ann Surg Oncol. 2009;16(10):2895-2907. doi:10.1245/ s10434-009-0548-8

10. Slater NJ, Montgomery A, Berrevoet F, et al. Criteria for definition of a complex abdominal wall hernia. Hernia. 2014;18(1):7-17. doi:10.1007/s10029-013-1168-6

11. Watson D. Current state of repair of large hiatal hernia. Int J Abdominal Wall Hernia Surg. 2019;2(2):39-43. doi:10.4103/ ijawhs.ijawhs_12_19

12. Mericli AF, Baumann DP, Butler CE. Reconstruction of the abdominal wall after oncologic resection: defect classification and management strategies. Plast Reconstr Surg. 2018;142(3 Suppl):187S-196S. doi:10.1097/PRS.0000000000004877

13. Bittner R, Bain K, Bansal VK, et al. Update of guidelines for laparoscopic treatment of ventral and incisional abdominal wall hernias (International Endohernia Society (IEHS))-Part A. Surg Endosc. 2019;33(10):3069-3139. doi:10.1007/s00464-019-06907-7

14. Bittner R, Bain K, Bansal VK, et al. Update of guidelines for laparoscopic treatment of ventral and incisional abdominal wall hernias (International Endohernia Society (IEHS)): part B. Surg Endosc. 2019;33(11):3511-3549. doi:10.1007/s00464-019-06908-6

15. Crago AM, Denton B, Salas S, et al. A prognostic nomogram for prediction of recurrence in desmoid fibromatosis. Ann Surg. 2013;258 (2):347-353. doi:10.1097/SLA.0b013e31828c8a30

16. Salas S, Dufresne A, Bui B, et al. Prognostic factors influencing progression-free survival determined from a series of sporadic desmoid tumors: a wait-and-see policy according to tumor presentation. $J$ Clin Oncol. 2011;29(26):3553-3558. doi:10.1200/JCO.2010.33.5489 


\section{Publish your work in this journal}

The International Journal of General Medicine is an international, peer-reviewed open-access journal that focuses on general and internal medicine, pathogenesis, epidemiology, diagnosis, monitoring and treatment protocols. The journal is characterized by the rapid reporting of reviews, original research and clinical studies across all disease areas. The manuscript management system is completely online and includes a very quick and fair peer-review system, which is all easy to use. Visit http://www.dovepress.com/ testimonials.php to read real quotes from published authors.

Submit your manuscript here: https://www.dovepress.com/international-journal-of-general-medicine-journal 